\title{
ESR1 Amplification in Breast Cancer by Optimized RNase FISH: Frequent but Low-Level and Heterogeneous
}

\author{
Cathy B. Moelans ${ }^{1 *}$, Frederik Holst ${ }^{2,4^{*} \odot}$, Olaf Hellwinkel ${ }^{3}$, Ronald Simon ${ }^{4}$, Paul J. van Diest ${ }^{1}$ \\ 1 Department of Pathology, University Medical Center Utrecht, Utrecht, The Netherlands, 2 Section of Gynecology and Obstetrics, Department of Clinical \\ Science, Haukeland University Hospital, Bergen, Norway, 3 Department of Legal Medicine, University Medical Center Hamburg Eppendorf, Hamburg, Germany, \\ 4 Department of Pathology, University Medical Center Hamburg Eppendorf, Hamburg, Germany
}

\begin{abstract}
Prevalence of ESR1 amplification in breast cancer is highly disputed and discrepancies have been related to different technical protocols and different scoring approaches. In addition, pre-mRNA artifacts have been proposed to influence outcome of ESR1 FISH analysis. We analyzed ESR1 gene copy number status combining an improved RNase FISH protocol with multiplex ligation-dependent probe amplification (MLPA) after laser microdissection. FISH showed a high prevalence of ESR1 gains and amplifications despite RNase treatment but MLPA did not confirm ESR1 copy number increases detected by FISH in more than half of cases. We suggest that the combination of the ESR1-specific intra-tumor heterogeneity and low-level copy number increase accounts for these discrepancies.
\end{abstract}

Citation: Moelans CB, Holst F, Hellwinkel O, Simon R, van Diest PJ (2013) ESR1 Amplification in Breast Cancer by Optimized RNase FISH: Frequent but Low-Level and Heterogeneous. PLoS ONE 8(12): e84189. doi:10.1371/journal.pone.0084189

Editor: Xin-Yuan Guan, The University of Hong Kong, China

Received August 26, 2013; Accepted November 13, 2013; Published December 18, 2013

Copyright: ( 2013 Moelans et al. This is an open-access article distributed under the terms of the Creative Commons Attribution License, which permits unrestricted use, distribution, and reproduction in any medium, provided the original author and source are credited.

Funding: This study was supported by the Deutsche Forschungsgemeinschaft (DGF SI 1347/3-1). The funders had no role in study design, data collection and analysis, decision to publish, or preparation of the manuscript.

Competing interests: The authors have declared that no competing interests exist.

*E-mail: C.B.Moelans@umcutrecht.nl (CBM); Frederik.Holst@ikm.uib.no (FH)

๑ These authors contributed equally to this work.

\section{Introduction}

Since the discovery of trastuzumab for treatment of HER2amplified breast cancer, fluorescence in-situ hybridization (FISH) based gene copy number analysis has become a standard tool in molecular pathology laboratories. While HER2 amplification is usually high-level, other clinically relevant genes may show only low-level copy number alterations, e.g. EGFR, HER3, and PIK3CA gene copy number alterations in lung cancer [1-11]. The ESR1 gene, encoding estrogen receptor alpha (ER), is probably the most disputed example of such low-level amplified genes. Since our initial report of $36 \%$ copy number increase [12], numerous studies have either confirmed [13] or challenged [14] that ESR1 is frequently gained in breast cancer. Discrepancies have been related to different copy number enumeration methods and scoring criteria while sensitivity challenges of non morphological methods using isolated DNA are still not fully addressed $[15,16]$. Further it was suggested that hybridization of the FISH probe to ESR1 pre-messenger RNA could result in aggregates of FISH signals which might be easily misinterpreted as gene amplification [17]. However, RNase treatment can impair FISH analysis by its DNA binding properties $[18,19]$. To estimate the influence of RNase treatment on ESR1 FISH analysis outcome, we developed an improved RNase FISH protocol permitting complete RNA elimination after pepsin digestion including a thorough RNase removal and, thus, a clean and clear gene copy number visualization. We analyzed breast cancer large sections and tissue microarrays (TMAs) using standard and RNase FISH protocols, and also employed multiplex ligationdependent probe amplification (MLPA) after laser-capture microdissection of tumor cells as an RNA-independent means of ESR1 copy number determination. Large sections were taken from 24 high-grade breast cancers with ESR1 amplification according to pre-existing FISH results from a "classical" FISH assay and a differential qRT-PCR approach using SOD2 and ESR2 as reference genes [12]. ESR1 copy numbers were re-assessed in 1-4 different cancerous areas in each of these 24 tumors (total: 50 areas) by a commercial FISH assay resulting in 15 tumors showing ESR1 amplification and 9 showing ESR1 gain, followed by a validation by MLPA and our improved RNase FISH analysis. In addition, a TMA of 147 consecutive breast cancers was analyzed for validation with the classical and the RNase FISH protocol. 


\section{Methods}

\section{Ethics statement}

All tissue samples included in this study were doublepseudomized left-over samples from pathological routine diagnosis in the Department of Pathology, University Medical Center Hamburg Eppendorf, Hamburg, Germany, that can be used for research without informed consent according to the laws of the State of Hamburg, Germany ( $\$ 12 \mathrm{HmbKHG}$ ). Manufacturing and usage of tissue microarrays for research purposes has been approved by the local Institutional Review Board (WF-049/09) of the Aerztekammer Hamburg (Chair: Prof. T. Weber).

\section{Patient material}

Out of a consecutive subset of 90 formalin fixed, paraffin embedded grade 3 breast cancers from the archives of the Department of Pathology in Hamburg that had been previously used in an ESR1 FISH mapping study [12], a total of 35 cases were selected including 24 tumors with increased ESR1 copy numbers by FISH (15 amplified and 9 gained) and 11 tumors without ESR 1 copy number increase. In most cases more than one tissue area was selected for FISH/MLPA analysis, resulting in all together 50 different tumor areas with and 19 areas without ESR1 copy number increase. 13 of the 24 tumors with and 10 of the 14 tumors without increased ESR1 copy number by FISH had previously been examined by TaqMan RT-qPCR using ESR2 and SOD2 as reference genes (primer and probe sequences see Table S3, method as previously described [12]). In addition, a tissue microarray (TMA) was constructed from 147 consecutive - but selected for availability of at least 8 tumor containing tissue blocks - breast tumors (14\% grade $1,57 \%$ grade 2 and $29 \%$ grade 3 ) and subjected to FISH analysis.

\section{FISH}

Standard ESR1 FISH analysis was performed using the ZytoLight ${ } S$ SPEC ESR1/CEN 6 Dual Color Probe Kit (Zytovision, Germany, Z-2070-20) according to the manufacturer's instructions with minor modifications. Briefly, slides were deparaffinized and incubated for 15 minutes in Heat Pretreatment Solution Citric at $98^{\circ} \mathrm{C}$. Slides were incubated in a pepsin solution for $10 \mathrm{~min}$ at $37^{\circ} \mathrm{C}$, washed in Wash buffer SSC, dehydrated and air dried. Subsequently, 10 $\mu \mathrm{l}$ of ZytoLight@SPEC ESR1/CEN 6 Dual Color Probe was applied to the slides followed by denaturation at $75^{\circ} \mathrm{C}$ for 10 min and incubation for $48-72$ hours at $37^{\circ} \mathrm{C}$ in a Thermobrite StatSpin system (Abbott Molecular). After hybridization, coverslips were removed in Wash Buffer $\mathrm{A}$ at $37^{\circ} \mathrm{C}$ for $2 \mathrm{~min}$, followed by wash steps in the same Wash Buffer for $2 \times 5$ min at $37^{\circ} \mathrm{C}$, dehydration. Slides were counterstained with DAPI/ Antifade solution for 15 min in the dark.

For FISH with RNase pretreatment, an additional RNase digestion step was introduced into the standard protocol after pepsin treatment (providing a better access of RNase to the gene locus than before pepsin digestion). Slides were rinsed in $2 \times$ SSC buffer and incubated with RNase A solution $(100 \mu \mathrm{g} /$ $\mathrm{ml}$ in $2 \times \mathrm{SSC}, \mathrm{pH} 7.4$, Macherey-Nagel) for $30 \mathrm{~min}$ at $37^{\circ} \mathrm{C}$.
Due to the binding affinity of RNase $A$ to (especially denatured single stranded) DNA, the DNA-bound enzyme blocks FISH probe binding to the target DNA and FISH signals are weakened or totally undetectable. To resolve the DNA-RNase complex an additional $\mathrm{NaCl}$ washing step was performed after RNase treatment $[18,19]$. Slides were rinsed $5 \mathrm{~min}$ in $2 \times$ SSC followed by 5 min washing in $0.1 \mathrm{M} \mathrm{NaCl}$ at room temperature, before the standard protocol was continued. Without the $0.1 \mathrm{M}$ $\mathrm{NaCl}$ washing step, interpretation of FISH results was hindered due to lack of signals of sufficient brightness and quality.

A strip protocol was used [20] to analyze the same tissue sections twice both with and without RNase treatment. Wash buffer ( $2 \times$ SSC, $0.3 \%$ NP 40 ) was added to release coverslips and remove $\mathrm{DAPI} / g$ lycerol mix and oil residues. After a short $2 \times$ SSC wash, slides were incubated with denaturation solution (70\% formamide / $2 \times$ SSC, $\mathrm{pH} 7.0-7.4$ ) at $73^{\circ} \mathrm{C}$ for $2 \times 2.5$ minutes to remove the hybridized probe. This was followed by a short 2× SSC washing step, incubation with RNase A and a $\mathrm{NaCl}$ washing step, according to the modified standard protocol. For EGFR FISH, a probe combination of EGFR (LSI EGFR SpectrumOrange, Vysis/Abbott) and centromere 7 (CEP 7 SpectrumGreen, Abbott) was used as previously described [11]. RNase A digestion consistently resulted in the disappearing of fuzzy signal clouds (like pre-mRNA appears [21-23]), while sharp point shaped signals (clusters) remained. This effect was interpreted as the different appearance of premRNA (fuzzy clouds) and DNA copy (point-shaped signals) mediated FISH signals due to pre-mRNA processing [24], and was taken as an internal control for complete RNA elimination. Evaluation of FISH copy numbers was carried out using a Zeiss Axio Imager A1 fluorescence microscope equipped with a Zeiss AxioCam and AxioVision imaging software. Analyzed whole sections and TMA spots where attentively manually scanned for copy number elevations. Copy number was determined by taking the full z-axis size of the analyzed nuclei into account [25]. In case of tumor heterogeneity, within the tumor region with the highest copy number increase by $\mathrm{FISH}$, 20 nuclei were randomly selected for copy number determination and used to define the amplification status of the according area. Interpretation was based on the average copy number ratio ESR1/CEN6 in 20 entire and non-overlapping nuclei. Applying FISH analysis without RNase treatment in tumors with cell nuclei showing tight as well as confluent signal clusters, the copy number was determined in nuclei with distinguishable signals. An average ratio $\geq 2$ was rated as amplification, and $\geq 1.3$ as gain [26]. Interpretation of $\mathrm{FISH}$ results after RNase treatment was blinded to FISH results without RNase treatment. Cases without an elevated copy number (ratio ESR1/CEN6 21.3) including normal (ratio ESR1/ CEN6 $=2 / 2$ ), low level gained (ratio ESR1/CEN6 <1.3), deleted and "polysomic" cases, were defined as "not increased".

\section{Laser microdissection}

Laser microdissection (PALM, Zeiss) was performed on 4 to six $3 \mu \mathrm{m}$ thick paraffin sections (by comparing with a serial H\&E stained slide where FISH amplified/non-amplified areas were marked). For laser microdissection, sections were baked at $56^{\circ} \mathrm{C}$ for 1 hour, deparaffinized in xylene for 10 minutes and 
rehydrated through graded alcohols $(100 \%, 85 \%$ and $70 \%$ for 1 minute each). After staining with haematoxylin for 5 seconds, slides were rinsed in water and dipped in eosin for 5 seconds. Finally, slides were dehydrated in $100 \%$ ethanol for 1 minute and air dried.

\section{Multiplex ligation-dependent Probe Amplification (MLPA)}

After laser microdissection, DNA was isolated by overnight incubation in proteinase $\mathrm{K}(10 \mathrm{mg} / \mathrm{ml}$; Roche, Almere, The Netherlands) at $56^{\circ} \mathrm{C}$ followed by heat inactivation for $10 \mathrm{~min}$. The DNA supernatant (30-50 $\mu \mathrm{l})$ was, after centrifugation, used in the MLPA analysis according the manufacturers' instructions, using the P078-B1 breast kit (MRC Holland, Amsterdam, The Netherlands) containing two ESR1 probes. All tests were performed in duplicate on an ABI 9700 PCR machine (Applied Biosystems, Foster City, CA, USA). PCR products were analysed on an ABI3730 DNA analyser (Applied Biosystems). Gene copy numbers were analyzed using Genemapper (Applied Biosystems) and Coffalyser (version 7.0) software (MRC-Holland). Six negative reference samples (4 blood samples and 2 normal breast samples) were taken along in each MLPA run to normalize MLPA ratios.

To avoid loss of sensitivity due to suboptimal probe sequence specific properties, results of both probes where compared by calculating the average MLPA ratio for each of the two MLPA ESR1 probes, based on 19 tumor areas showing no copy number increase by FISH (average ESR1 copy number: 1.95, average ESR1/CEP6 ratio: 0.99). The first probe showed an average MLPA copy number ratio of 1.01 and thus an almost exact normalization whereas the second MLPA probe showed a ratio of only 0.83 indicating a less efficient amplification leading to a significant difference in copy number ratio between both probes $(p<0.001)$. Therefore, only the first MLPA probe was used for further calculations and comparisons. An MLPA ratio value below 0.7 was defined as loss, a value between 0.7-1.3 was defined as normal, 1.3-2.0 as gain, and values $>2.0$ were defined as (high level) amplification, as established previously [27-30]. Interpretation of MLPA was done blinded to FISH results. Positive MLPA results (ratio 21.3 ) were matched to previously existing FISH mapping data [13]: 8 (of 16) cases with MLPA copy number elevations were predominantly limited to the ESR1 gene only whereas 6 (of 8 ) cases showed more extended amplicons, (see Table S1).

\section{Statistics}

Statistics were performed using SPSS statistical software (15.0). ER en PR protein status were determined according to Remmele et al. [31], with a score $>2$ regarded as positive. Differences in FISH, MLPA or qPCR copy number ratios were evaluated using the Mann-Whitney $U$ test (unpaired) or Wilcoxon signed rank test (paired). Correlation between FISH and MLPA was calculated using the Spearman rho correlation coefficient. Two-sided p-values $<0.05$ were defined as significant. Figures were made using GraphPad Prism 5.

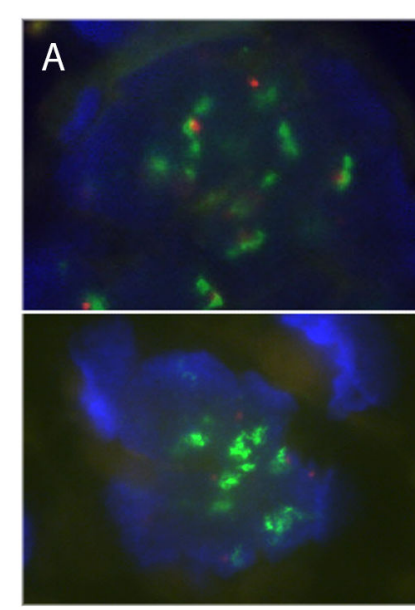

B

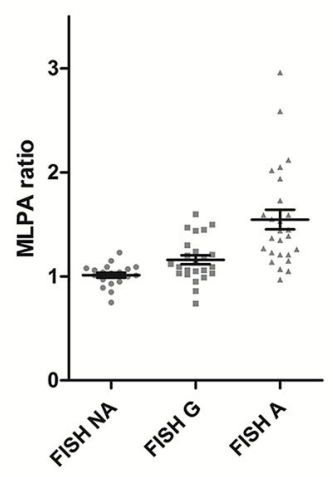

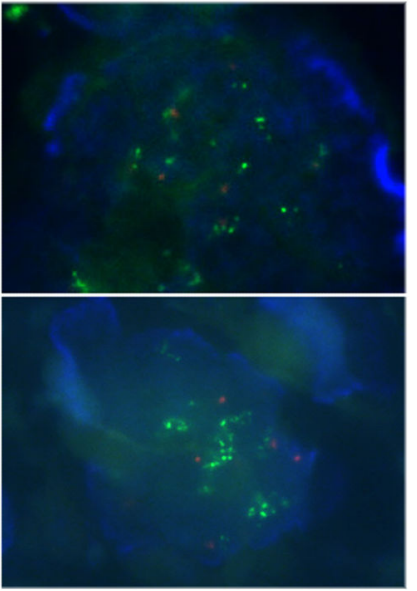

RNAse

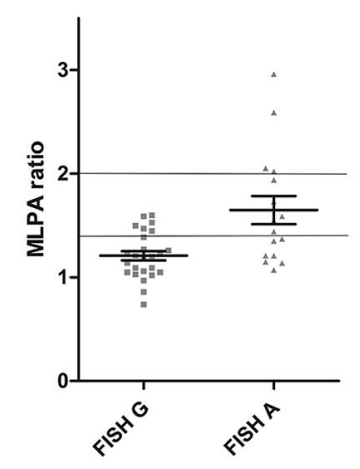

Figure 1. Effect of RNase treatment on FISH and on correlation between FISH and MLPA. (a) RNase pretreatment resulted in a higher fraction of tumor cells showing point-shaped FISH signals, by eliminating eye catching fuzzy clusters of ESR1 signals seen by standard FISH (b) MLPA copy number ratios in FISH "not increased", gained and amplified samples without and with RNase treatment. doi: 10.1371/journal.pone.0084189.g001

\section{Results}

\section{Effect of RNase treatment on FISH copy numbers and signal size and shape}

RNase pretreatment resulted in a higher fraction of tumor cells showing point-shaped FISH signals, by eliminating fuzzy clusters (fringes, tails or clouds) of ESR1 FISH probe signals (Figure 1) seen in many nuclei by standard FISH.

But FISH with and without RNase treatment showed no major differences in gene copy number, neither in the whole sections, nor in the TMA format. In the 50 whole section areas of 24 breast carcinomas with increased copy number, the average ESR1 copy number was 4.55 (range $2.30-12.05$ ) preand 4.44 (range: $2.10-13.70)$ post-RNase $(p=0.47)$ and the average ESR1/CEP6 ratio was 2.16 (range 1.28-6.89) pre- and 1.98 (range $1.13-5.37)$ post-RNase $(p=0.006)$. Accordingly, the amplification status remained unchanged in 12/15 tumors (cut- 
off ratio 2.0) after RNase treatment, whereas three cases were down-staged to gains (cut-off ratio 1.3). 7/9 FISH-gained cases remained gained after RNase treatment, one case was reclassified as amplified and the other as not increased (cut-off ratio 1.3). To illustrate signal appearance, all 24 cases with whole sections are documented with and without RNase treatment in a supplementary photographical dataset (Optical Dataset S1 and S2)

In the TMA, 82/147 tumor spots were analyzable by classical and RNase FISH. Without RNase, 32 (39\%) tumors showed an increased ESR1 copy number including 11 (13\%) amplifications and $21(26 \%)$ gains. After RNase treatment, 30 $(37 \%)$ tumors showed increased ESR1 copy numbers (13 (16\%) amplifications and $17(21 \%)$ gains), while the ESR1 status changed from "gain" to "not increased" in two, from "amplification" to "gain" in one, and from "gain" to "amplification" in three tumors (Table S2). The average ESR1 copy number for the 32 cases considered "ESR1 copy number increased" pre-RNase was 3.39 pre- and 3.49 post-RNase $(p=0.558)$, and the average ESR $1 / C E P 6$ ratio was 1.90 (range 1.31-3.08) pre- and 2.05 (range 1.15-4.16) post-RNase $(p=0.83)$.

Taken together, in $94.6 \%$ of tumors with increased ESR1 gene copy number before RNase treatment, the ESR1 status remained increased (mostly cluster type including gene dupliand triplication $[9,32,33]$ ) after RNase treatment. Interestingly, a commercially available EGFR FISH probe (Abbott) showed the same RNase sensitive changes of signal appearance as the ESR1 probe in this study (see Figure S1).

\section{Effect of RNase treatment on correlation between FISH and MLPA}

Tumors with and without ESR1 copy number increase by FISH showed significant differences by MLPA and qPCR regardless of the assay used (qPCR ESR1/ESR2 $p=0.002$; qPCR ESR1/SOD2 $p=0.010$; MLPA $p=0.011$ ), although $q P C R$ ratios after $E S R 2$ normalization were significantly higher than after SOD2 normalization $(p<0.0001)$.

MLPA ratios were highly correlated with FISH ratios regardless of whether FISH was performed with or without RNase pretreatment. Tumors that were rated gained or amplified by FISH had significantly higher MLPA ratios than those with "not increased" FISH findings $(p=0.0005$ for gain and $p=0.0125$ for amplification without RNase; $p<0.0001$ for gain and $p=0.0041$ for amplification with RNase; Figure 1). Spearman rho correlation between MLPA and FISH in cases with elevated copy numbers (gains and amplifications) by $\mathrm{FISH}$, prior and after RNase treatment, was 0.57 (0.35-0.73) and $0.56(0.31-0.74)$, respectively (both $p<0.0001)$. The overall correlation between FISH with and without RNase was 0.78 (0.62-0.88; $p<0.0001$ ) (see Figure S2). 54\% (13/24) of FISHincreased tumors without RNase treatment, and $57 \%(13 / 23)$ of FISH-increased tumors after RNase treatment failed to yield increased copy numbers by MLPA. Although the fraction of "MLPA failures" was highest (7/10) for tumors with gains by $\mathrm{FISH}$, there were also $3 / 13$ tumors with apparently homogeneous and high-level FISH amplification (for example case 7 and 16 from Table S1) that failed to show an ESR1 copy number increase by MLPA. FISH positive but MLPA negative samples had a significantly lower FISH copy numbers than MLPA positive samples (1.79 vs. 2.37 after RNase; $p=0.009$ ), as well as a higher rate of heterogeneity (83\% (19 of 23 cases) vs. $41 \%$ ( 7 of 17 cases); $p=0.007$ ), and averaged a significantly higher MLPA ratio compared to $\mathrm{FISH}$ negative cases (1.11 vs. 1.01, $p=0.015$ ) (Figure 1).

\section{Heterogeneity}

To take influence of heterogeneity into account, 70 different areas selectively identified by FISH analysis from 20 cancers were microdissected and subjected to MLPA analysis. As illustrated in Table S1, MLPA yielded considerably heterogeneous results from different areas of the same tumor block as well as between different tumor blocks of the same cancer specimen. Of all cases with multiple tumor blocks and/or areas analyzed, 25\% (5/20) showed a different copy number status by MLPA between or within blocks, and $39 \%$ (7/18) and $67 \%(8 / 12)$ by FISH without and with RNase, respectively. The average copy number ratio variation between/within tumor blocks was $0.55 / 0.56$ for $\mathrm{FISH}+$ RNase, and 0.25 for MLPA. Furthermore, particularly challenging for copy number determination in tumors, mosaic heterogeneity (cell-to-cell variation)- a well-known phenomenon for many genes [34-36] - was frequently (60\% of tumors in our study) observed, mostly in case of low copy number levels.

\section{Discussion}

In tumors with increased ESR1 copy number previously determined by a classical FISH assay, RNase A digestion prior to ESR1 FISH eliminated cloudy signal clusters caused by premRNA artifacts, resulting in clearly distinguishable signals, mostly of cluster (HSR) type. This phenomenon may not be limited to ESR1 FISH analysis, since similar artifacts were seen also in a case of EGFR-amplified lung cancer. These RNAinduced artifacts may pose a problem in particular for the interpretation of low-level amplified genes including ESR1, where only few extra gene copies determine the amplification status. However, in our study, RNase digestion did not significantly change the observed ESR1 gene copy number results. Overall, the fraction of tumors with elevated ESR1 copy numbers changed only slightly from $39 \%$ to $37 \%$. Especially as $\mathrm{FISH}$ analysis is the subjective interpretative translation of optical patterns into numerical information, these data suggest that the inter- and intra-observer differences on ESR1 copy number assessment and different analysis approaches (such as z-stack layers taken into account [25,37], number of countable nuclei considered sufficient for analyzability [38], selected tissue areas chosen, cut-offs used for status definition $[17,26,37,38]$ or even accepted signal quality) may be more influential than removal of pre-messenger RNA itself. In fact, in our hands, RNase pretreatment resulted in a higher fraction of tumor cells showing point-shaped FISH signals, by prohibiting eye catching pre-mRNA artifacts (Figure 1) seen in many nuclei by standard FISH, in line with the observations of Ooi. et al [17]. Nevertheless, it was possible to reliably determine ESR1 copy numbers in all large sections also without RNase 
treatment, since sufficient numbers of nuclei showed distinct and countable signals (see Optical Dataset S1 and S2).

While Ooi et al. reported a gain frequency of $5.9 \%(3 / 51)$ in breast carcinoma using FISH after RNase pre-treatment, the findings of the present study give evidence for copy number increase in $37 \%$ of cases. This large difference could be related to several factors. First, in contrast to Ooi et al [23] (personal communication), we used a different RNase treatment protocol where RNase digestion was performed after pepsin digestion and an additional $\mathrm{NaCl}$ step was introduced to eliminate DNA bound RNase. In addition we used a different FISH assay. Both could have significant influence on signal appearance. Second, we used full section tissue slides and a TMA (FFPE) instead of core needle biopsies (FFPE) and touch smears, and a different (subjective) way of analysis. For example, Ooi et al. used the HER2 ASCO criteria for evaluation of ESR1 (1.8 and 2.2 cut-offs) whereas we used 1.3 and 2.0 as cut-off. Lastly, the choice of study population may also be of significant influence, as e.g. in early breast cancer ESR1 amplification was detected at a lower frequency (14\% of ER positive cases in BIG 1-98 trial) [39] compared to advanced stage breast cancer $(23 \%$ revealed by the same $\mathrm{FISH}$ technique) [26].

Independent from RNase treatment, there was a significant difference in MLPA copy number ratio between samples that were FISH amplified and samples that showed only gain or no ESR1 copy number increase, although MLPA did not confirm ESR1 copy number increases detected by FISH in more than half of cases. Nevertheless this discrepancy was independent from RNase treatment, suggesting that reasons other than RNA-related hybridization artifacts account for the differences in ESR1 copy number alterations observed with different methods.

This finding either suggests false-positive FISH findings independently from RNase treatment, or, and probably more likely, that MLPA is not capable of identifying all tumors with increased ESR1 copy numbers due to threshold and dilution issues. As expected, different areas of one tumor showed different copy numbers by FISH as well as by MLPA. Of all tumors with multiple areas analyzed, $25 \%$ showed a different copy number status by MLPA between areas, and $39 \%$ and $67 \%$ by FISH without and with RNase, respectively. Revealingly, the FISH positive but MLPA negative samples had a significantly lower FISH copy number than MLPA positive cases and averaged a significantly higher MLPA ratio compared to FISH negative cases. Furthermore, FISH positive but MLPA negative samples had a higher rate of heterogeneity.

Since all tumor samples had been laser capture microdissected to minimize the influence of contaminating nonneoplastic cells, we therefore hypothesize that the low-level of ESR1 copy number changes and copy number heterogeneity could have obscured detection of ESR1 amplification by MLPA (e.g. in samples 5, 9 and 26, see Table S1).

Furthermore different efficiency of probe amplification (average MLPA ESR1/CEP6 ratio of 0.99 vs 0.83 ) and qPCR assay ratios $(p<0.0001)$ indicate that probe design and normalization may significantly influence the results of such
DNA quantifying methods, which is especially important in case of values around the threshold.

In summary these observations provide a probable explanation for the discrepant results that have been reported by ESR1 copy number assays using isolated DNA (such as MLPA), FISH and $\mathrm{CISH}$.

\section{Supporting Information}

Figure S1. A commercially available EGFR FISH probe (Abbott) showed the same RNase sensitive changes as the ESR1 probe in this study.

(JPG)

Figure S2. Correlation between MLPA and FISH. (JPG)

Table S1. MLPA results and FISH results without and with RNase treatment, performed on $\mathbf{3 5}$ breast tumors. (XLSX)

Table S2. FISH results without and with RNase treatment, performed on a breast TMA.

(XLSX)

Table S3. TaqMan RT-qPCR Primer and Reporter Sequences.

(DOC)

Optical Dataset S1. Optical dataset illustrating FISH signal appearance containing full size ESR1 FISH photos (not all of them representative) of a total of 35 breast cancer cases (Case\#1-35) including 24 tumours with increased ESR1 copy numbers by FISH (15 ESR1 amplified and 9 ESR1 gained, Case\#01-\#24) and 11 tumours without ESR1 copy number increase (Case\#25-\#35), selected out of a consecutive subset of 90 formalin fixed, paraffin embedded grade 3 breast cancers from the archives of the Department of Pathology in Hamburg that had been previously used in an ESR1 FISH mapping study [12]. For all 24 cases with ESR 1 copy number increase different pictures (Fig.01- ...) without RNase A treatment are available. The pictures document by three color photographs the ESR1 FISH appearance of nuclei with ESR1 copy number increase and nuclei without $E S R 1$ copy number increase on $4 \mu \mathrm{m}$ full section FFPE tissue slides, showing ESR1 signals (green), CEN6 signals (orange) and nuclei (blue) in $100 x$ or $63 x$ magnification. Pictures are subscribed "CNI" for observed gene "copy number increase" and "NO" for "normal" or "no copy number increase". Gene loci with additional allelic copies (CNI) are marked exemplarily in some cases (white arrows and edges). Especially if nuclei with "CNI" occur in a pattern of mosaic heterogeneity intermingled with nuclei without "CNI". Due to intensity variations and the three dimensional distribution of signals not all present gene copies can be shown. To illustrate the distribution of signals within the z-axis, some pictures are taken with two different z-layers (Z-Stack A-D). To illustrate the 
difference between cases (with tissue areas) showing ESR1 copy number increase (CNI) and cases without ESR1 copy number increase, pictures of the 11 cases with clearly "normal" copy number or "no copy number increase" ("NO") are shown in addition.

(DOCX)

Optical Dataset S2. Optical dataset illustrating FISH signal appearance containing full size ESR1 FISH photos (not all of them representative) of a total of 35 breast cancer cases (Case\#1-35) including 24 tumours with increased ESR1 copy numbers by FISH (15 ESR1 amplified and 9 ESR1 gained, Case\#01-\#24) and 11 tumours without ESR1 copy number increase (Case\#25-\#35), selected out of a consecutive subset of 90 formalin fixed, paraffin embedded grade 3 breast cancers from the archives of the Department of Pathology in Hamburg that had been previously used in an ESR1 FISH mapping study [12]. For all 24 cases with ESR 1 copy number increase different pictures (Fig.01- ...) with RNase A treatment are available. The pictures document by three color photographs the ESR1 FISH appearance of nuclei with ESR1 copy number increase and nuclei without ESR1 copy number increase on $4 \mu \mathrm{m}$ full section FFPE tissue slides, showing ESR1 signals (green), CEN6 signals (orange) and nuclei (blue) in 100x or 63x magnification. Pictures are subscribed "CNI" for observed gene "copy number increase" and "NO" for "normal" or "no copy number increase".

\section{References}

1. Ålgars A, Lintunen M, Carpén O, Ristamäki R, Sundström J (2011) EGFR gene copy number assessment from areas with highest EGFR expression predicts response to anti-EGFR therapy in colorectal cancer. Br J Cancer 105: 255-262. doi:10.1038/bjc.2011.223. PubMed: 21694725.

2. Cappuzzo F, Hirsch FR, Rossi E, Bartolini S, Ceresoli GL et al. (2005) Epidermal growth factor receptor gene and protein and gefitinib sensitivity in non-small-cell lung cancer. J Natl Cancer Inst 97: 643-655. doi:10.1093/jnci/dji112. PubMed: 15870435.

3. Cappuzzo F, Toschi L, Domenichini I, Bartolini S, Ceresoli GL et al. (2005) HER3 genomic gain and sensitivity to gefitinib in advanced nonsmall-cell lung cancer patients. $\mathrm{Br} \mathrm{J}$ Cancer 93: 1334-1340. doi: 10.1038/sj.bjc.6602865. PubMed: 16288303

4. Cappuzzo F, Varella-Garcia M, Shigematsu H, Domenichini I, Bartolini $S$ et al. (2005) Increased HER2 gene copy number is associated with response to gefitinib therapy in epidermal growth factor receptorpositive non-small-cell lung cancer patients. J Clin Oncol 23: 5007-5018. doi:10.1200/JCO.2005.09.111. PubMed: 16051952.

5. Dahabreh IJ, Linardou H, Kosmidis P, Bafaloukos D, Murray S (2011) EGFR gene copy number as a predictive biomarker for patients receiving tyrosine kinase inhibitor treatment: a systematic review and meta-analysis in non-small-cell lung cancer. Ann Oncol 22: 545-552. doi:10.1093/annonc/mdq432. PubMed: 20826716.

6. Dahabreh IJ, Linardou H, Siannis F, Kosmidis P, Bafaloukos D et al. (2010) Somatic EGFR mutation and gene copy gain as predictive biomarkers for response to tyrosine kinase inhibitors in non-small cell lung cancer. Clin Cancer Res 16: 291-303. doi: 10.1158/1078-0432.CCR-09-1660. PubMed: 20028749.

7. Fidler MJ, Morrison LE, Basu S, Buckingham L, Walters K et al. (2011) PTEN and PIK3CA gene copy numbers and poor outcomes in nonsmall cell lung cancer patients with gefitinib therapy. Br J Cancer 105: 1920-1926. doi:10.1038/bjc.2011.494. PubMed: 22095222.

8. Jensen KC, Turbin DA, Leung S, Miller MA, Johnson K et al. (2008) New cutpoints to identify increased HER2 copy number: analysis of a large, population-based cohort with long-term follow-up. Breast Cancer Res Treat 112: 453-459. doi:10.1007/s10549-007-9887-y. PubMed: 18193353.
Gene loci with additional allelic copies (CNI) are marked exemplarily in some cases (white arrows and edges). Especially if nuclei with "CNI" occur in a pattern of mosaic heterogeneity intermingled with nuclei without "CNI". Due to intensity variations and the three dimensional distribution of signals not all present gene copies can be shown. To illustrate the distribution of signals within the z-axis, some pictures are taken with two different $z$-layers (Z-Stack A-D). To illustrate the difference between cases (with tissue areas) showing ESR1 copy number increase (CNI) and cases without ESR1 copy number increase, pictures of the 11 cases with clearly "normal" copy number or "no copy number increase" ("NO") are shown in addition.

(DOCX)

\section{Acknowledgements}

We thank Sylvia Schnöger, Sascha Eghdessadi and Bianca Kelp for excellent technical support.

\section{Author Contributions}

Conceived and designed the experiments: PvD RS. Performed the experiments: CBM $\mathrm{FH} \mathrm{OH}$. Analyzed the data: $\mathrm{CM} \mathrm{FH}$. Contributed reagents/materials/analysis tools: CM FH RS PvD. Wrote the manuscript: $\mathrm{CM} \mathrm{FH}$.

9. Martin V, Mazzucchelli L, Frattini M (2009) An overview of the epidermal growth factor receptor fluorescence in situ hybridisation challenge in tumour pathology. J Clin Pathol 62: 314-324. doi:10.1136/ jcp.2008.059592. PubMed: 19052028.

10. Ribeiro FR, Henrique R, Martins AT, Jerónimo C, Teixeira MR (2007) Relative copy number gain of MYC in diagnostic needle biopsies is an independent prognostic factor for prostate cancer patients. Eur Urol 52: 116-125. doi:10.1016/j.eururo.2006.09.018. PubMed: 17070983.

11. Woelber L, Hess S, Bohlken H, Tennstedt P, Eulenburg C et al. (2012) EGFR gene copy number increase in vulvar carcinomas is linked with poor clinical outcome. J Clin Pathol 65: 133-139. doi:10.1136/ jcp-2010-079806. PubMed: 22128196.

12. Holst F, Stahl PR, Ruiz C, Hellwinkel O, Jehan Z et al. (2007) Estrogen receptor alpha (ESR1) gene amplification is frequent in breast cancer. Nat Genet 39: 655-660. doi:10.1038/ng2006. PubMed: 17417639.

13. Holst F, Moelans CB, Filipits M, Singer CF, Simon R et al. (2012) On the evidence for ESR1 amplification in breast cancer. Nat Rev Cancer 12: 149. doi:10.1038/nrc3093-c3. PubMed: 22270954.

14. Thomas C, Gustafsson JA (2011) Not enough evidence to include ESR1 amplification. Nat Rev Cancer 11: 823. doi:10.1038/nrc3093-c1. PubMed: 22020208

15. Teo SM, Pawitan Y, Ku CS, Chia KS, Salim A (2012) Statistical challenges associated with detecting copy number variations with nextgeneration sequencing. Bioinformatics 28: 2711-2718. doi:10.1093/ bioinformatics/bts535. PubMed: 22942022.

16. Treangen TJ, Salzberg SL (2012) Repetitive DNA and next-generation sequencing: computational challenges and solutions. Nat Rev Genet 13: 36-46. PubMed: 22124482.

17. Ooi A, Inokuchi M, Harada S, Inazawa J, Tajiri R et al. (2012) Gene amplification of ESR1 in breast cancers--fact or fiction? A fluorescence in situ hybridization and multiplex ligation-dependent probe amplification study. J Pathol 227: 8-16. doi:10.1002/path.4064. PubMed: 22170254

18. Jensen DE, von Hippel PH (1976) DNA "melting" proteins. I. Effects of bovine pancreatic ribonuclease binding on the conformation and stability of DNA. J Biol Chem 251: 7198-7214. PubMed: 993211. 
19. Felsenfeld G, Sandeen G, Vonhippel PH (1963) The Destabilizing Effect of Ribonuclease on the Helical DNA. Structure - Proc Natl Acad Sci U S A 50: 644-651. doi:10.1073/pnas.50.4.644

20. Epstein L, DeVries S, Waldman FM (1995) Reutilization of previously hybridized slides for fluorescence in situ hybridization. Cytometry 21: 378-381. doi:10.1002/cyto.990210410. PubMed: 8608736.

21. Xing Y, Johnson CV, Moen PT Jr., McNeil JA, Lawrence J (1995) Nonrandom gene organization: structural arrangements of specific premRNA transcription and splicing with SC-35 domains. J Cell Biol 131: 1635-1647. doi:10.1083/jcb.131.6.1635. PubMed: 8557734.

22. Mühlemann O, Mock-Casagrande CS, Wang J, Li S, Custódio N et al. (2001) Precursor RNAs harboring nonsense codons accumulate near the site of transcription. Mol Cell 8: 33-43. doi:10.1016/ S1097-2765(01)00288-X. PubMed: 11511358.

23. Johnson C, Primorac D, McKinstry M, McNeil J, Rowe D et al. (2000) Tracking COL1A1 RNA in osteogenesis imperfecta. splice-defective transcripts initiate transport from the gene but are retained within the SC35 domain. J Cell Biol 150: 417-432. doi:10.1083/jcb.150.3.417. PubMed: 10931857

24. Smith KP, Moen PT, Wydner KL, Coleman JR, Lawrence JB (1999) Processing of endogenous pre-mRNAs in association with SC-35 domains is gene specific. J Cell Biol 144: 617-629. doi:10.1083/jcb. 144.4.617. PubMed: 10037785.

25. Tomita S, Zhang Z, Nakano M, Ibusuki M, Kawazoe T et al. (2009) Estrogen receptor alpha gene ESR1 amplification may predict endocrine therapy responsiveness in breast cancer patients. Cancer Sci 100: 1012-1017. doi:10.1111/j.1349-7006.2009.01145.x. PubMed: 19320640.

26. Laenkholm AV, Knoop A, Ejlertsen B, Rudbeck T, Jensen MB et al. (2012) ESR1 gene status correlates with estrogen receptor protein levels measured by ligand binding assay and immunohistochemistry. Mol Oncol 6: 428-436. doi:10.1016/j.molonc.2012.04.003. PubMed: 22626971.

27. Coffa J, van de Wiel MA, Diosdado B, Carvalho B, Schouten J et al. (2008) MLPAnalyzer: data analysis tool for reliable automated normalization of MLPA fragment data. Cell Oncol 30: 323-335. PubMed: 18607067.

28. Bunyan DJ, Eccles DM, Sillibourne J, Wilkins E, Thomas NS et al. (2004) Dosage analysis of cancer predisposition genes by multiplex ligation-dependent probe amplification. $\mathrm{Br} \mathrm{J}$ Cancer 91: 1155-1159. doi: 10.1038/sj.bjc.6602121. PubMed: 15475941

29. Vorstman JA, Jalali GR, Rappaport EF, Hacker AM, Scott C et al. (2006) MLPA: a rapid, reliable, and sensitive method for detection and analysis of abnormalities of 22q. Hum Mutat 27: 814-821. doi:10.1002/ humu.20330. PubMed: 16791841.

30. Moelans CB, de Weger RA, van Diest PJ (2010) Multiplex ligationdependent probe amplification to detect HER2 amplification in breast cancer: new insights in optimal cut-off value. Cell Oncol 32: 311-312. PubMed: 20442492

31. Remmele W, Stegner HE (1987) Recommendation for uniform definition of an immunoreactive score (IRS) for immunohistochemical estrogen receptor detection (ER-ICA) in breast cancer tissue. Pathologe 8: 138-140. PubMed: 3303008.

32. Trask BJ (2002) Human cytogenetics: 46 chromosomes, 46 years and counting. Nat Rev Genet 3: 769-778. doi:10.1038/nrg905. PubMed: 12360235.

33. Braude I, Vukovic B, Prasad M, Marrano P, Turley S et al. (2006) Large scale copy number variation (CNV) at $14 q 12$ is associated with the presence of genomic abnormalities in neoplasia. BMC Genomics 7: 138. doi:10.1186/1471-2164-7-138. PubMed: 16756668.

34. Szollosi J, Balazs M, Feuerstein BG, Benz CC, Waldman FM (1995) ERBB-2 (HER2/neu) gene copy number, p185HER-2 overexpression, and intratumor heterogeneity in human breast cancer. Cancer Res 55: 5400-5407

35. Bernasconi B, Chiaravalli AM, Finzi G, Milani K, Tibiletti MG (2012) Genetic heterogeneity in HER2 testing may influence therapy eligibility. Breast Cancer Res Treat 133: 161-168. doi:10.1007/ s10549-011-1744-3. PubMed: 21901388.

36. Snuderl M, Fazlollahi L, Le LP, Nitta M, Zhelyazkova BH et al. (2011) Mosaic amplification of multiple receptor tyrosine kinase genes in glioblastoma. Cancer Cell 20: 810-817. doi:10.1016/j.ccr.2011.11.005. PubMed: 22137795

37. Pentheroudakis G, Kotoula V, Eleftheraki AG, Tsolaki E, Wirtz RM et al. (2013) Prognostic Significance of ESR1 Gene Amplification, mRNA Protein Expression and Functional Profiles in High-Risk Early Breast Cancer: A Translational Study of the Hellenic Cooperative Oncology Group (HeCOG). PLOS ONE 8: e70634. doi:10.1371/journal.pone. 0070634 . PubMed: 23923010.

38. Lin CH, Liu JM, Lu YS, Lan C, Lee WC et al. (2013) Clinical significance of ESR1 gene copy number changes in breast cancer as measured by fluorescence in situ hybridisation. J Clin Pathol 66: 140-145. doi:10.1136/jclinpath-2012-200929. PubMed: 23268322

39. Ejlertsen B, Aldridge J, Nielsen KV, Regan MM, Henriksen KL et al. (2012) Prognostic and predictive role of ESR1 status for postmenopausal patients with endocrine-responsive early breast cancer in the Danish cohort of the BIG 1-98 trial. Ann Oncol 23: 1138-1144. doi:10.1093/annonc/mdr438. PubMed: 21986093. 